\title{
Gender Differences In Web Navigation
} Strategies, Efficiency \& Confidence

\author{
SHARON MCDONALD and LINDA SPENCER \\ School of Computing, Engineering and Technology, University of Sunderland, \\ sharon.mcdonald@sunderland.ac.uk, linda.spencer@sunderland.ac.uk
}

Abstract: This paper presents the results of the first study in a series of experiments that were designed to examine gender differences in web navigation. In this study participants were asked to locate the answers to ten questions via navigation through a large university website. A variety of behavioural and self-report measures were used to examine gender differences in navigational efficiency, navigational strategy and user confidence. The results show that although there were no overall differences between males and females in terms of navigational efficiency, male participants expressed a significantly greater degree of confidence in their ability to navigate through the web than female participants. The results also show that gender differences are apparent in the types of navigational strategies used, with female participants relying predominately on landmark information to guide their navigational choices. These results are discussed in relation to their implications for the design of web-based navigational aids that support the differing navigational strategies favoured by male and female users.

\section{INTRODUCTION}

Although the web is rapidly becoming the world's dominant global information resource, there is evidence to suggest that users are unable to explore navigable hypertext-based interfaces such as the web without experiencing disorientation [1, 2, 3]. Moreover, evidence from research into navigation in real and electronic worlds suggests that gender related differences could seriously impact upon navigation in hypertext environments such as the web. For example, studies have shown that males demonstrate more efficient navigation behaviour and higher levels of 
confidence in their ability to navigate through unfamiliar environments than females in both real and virtual worlds [4]. Studies have also shown that males and females rely upon different factors within the environment to guide their navigation [5]. Clearly, findings such as these have implications for the design of navigable systems, and the navigational aids that support exploration of those systems by both males and females.

The aim of the experiment presented here was to examine gender differences in user confidence, efficiency and strategy in web navigation.

\section{NAVIGATION IN REAL AND ELECTRONIC WORLDS}

Until the mid 1980s, navigation research focused largely on real world environments; however with the growing popularity of navigable interfaces such as hypertext, the question of how people navigate through complex electronic spaces became an issue for research. The majority of these studies report that users experience the same types of navigational problems as they might encounter when navigating through an unfamiliar real world environment [6]. This observation has led researchers to suggest clear parallels between navigation in physical and electronic space.

As yet there have been no attempts to study the impact of gender differences on navigation in hypertext. However, experiments designed to study navigation in the real world and in computer simulations have shown that gender differences are apparent in navigation efficiency [4], chosen navigational strategy $[5,7,8,9]$ and in the degree of confidence expressed by the navigator [4,9]. For example, Schmitz [9] found that girls expressed a greater degree of anxiety than boys when completing a real world walk through maze task, and that girls relied upon salient landmarks within the maze to guide their navigation to a greater extent than boys.

The experimental hypothesis was that gender related differences apparent in studies of real world navigation would also occur in navigation of electronic spaces such as the web.

\section{METHOD}

\subsection{Participants}

Twenty volunteers participated in this study, ten males and ten females, all of the participants were 16 years of age, and had equivalent levels of 
experience with computers and the World Wide Web. Participants were from similar educational backgrounds.

\subsection{Materials and Design}

The University of Sunderland's School of Computing, Engineering and Technology's web-site (http://osiris.sunderland.ac.uk) was used in the experiment. The site provides information for staff, students and prospective students on all aspects of the department. The site is connected to the University of Sunderland's main homepage and has a large number of external and internal hyper-links. The basic topology of the site is a mixed structure comprising a basic hierarchical frame with a number of crossreferential links. The site was developed over a period of five years and is periodically reviewed and evaluated in terms of both the accuracy of the information presented and the usability of the site. The site was viewed via Netscape Communicator 4.6.

The experiment used a between subjects (Independent groups) design. The independent variable was gender (male vs. female). The dependent variables were measures of navigation efficiency, navigation strategies and user confidence.

\subsection{Procedure}

The experiment was conducted using two distinct tasks, a search task and a direction-giving task. Before beginning the experimental tasks, participants were given five minutes to become acquainted with the website.

The first experimental task required participants to navigate through the website to locate the answers to ten questions. The answer to each question could be found on one particular page in the site. Participants were instructed to find each answer by following the most direct path; access to search facilities was denied. The number of correctly answered questions was recorded, and the number of additional nodes opened was recorded for each participant. Specifically, the shortest number of nodes to each answer was calculated, this was then subtracted from the actual number of nodes opened (excluding backtracks) to give an additional node score. Participants began each search from the top-level entry node in Figure 1.

After each completed search participants were required to make several ratings regarding their confidence levels while performing the search task. Specifically, subjects were asked to rate on a five-point scale ranging from Strongly Agree to Strongly Disagree the extent to which they agreed with two statements. Statement 1 referred to how confident they were that the answers they gave were correct. Statement 2 referred to their confidence in 
their ability to navigate efficiently through the web-site. At this point, participants were also asked to indicate whether they had located the answers by following a planned route, or whether they had reacted to cues within the web-site, such as landmarks.

The second experimental task was a direction-giving task. This task was included in the experiment in order to obtain verbal protocol data regarding the users' navigational strategies. We were particularly interested in comments about wayfinding decisions and landmarks. Participants were asked to direct a co-experimenter to a specified area within the web-site.

Verbal protocols were recorded and analysed in the following way. The verbal protocols were analysed for references to landmarks within the site and for comments concerning wayfinding decision making. Comments about landmarks were categorised according to whether the landmark was a) used to make navigational decisions; b) used as a navigational goal; or c) recognised from a previous encounter. Comments about wayfindng decisions were categorised as a) definite action, e.g. follow this link; b) casual analysis, e.g. let me think; or c) exploratory action, e.g. try this.

It was decided to obtain verbal protocol data in this manner rather than asking users to verbalise their thoughts during the search task, The technique known as Constructive Interaction [10] was used so that some of the problems associated with the use of verbal protocols could be overcome.

\section{RESULTS}

\subsection{Confidence}

After each completed search participants were asked to rate the extent to which they agreed or disagreed with the two statements below.

Statement 1: I was confident that the answers I gave were correct.

An independent samples Mann Whitney $U(Z=-3.50, p<0.00)$ test revealed that the male (mean rating 4.4) participants agreed with this statement to a greater extent than the female participants (mean rating 3.7), indicating that they felt more confident that the answers they gave in response to the questions were correct.

Statement 2: I was confident that I followed the most accurate (shortest) route to the information I required.

An independent samples Mann Whitney $U(Z=-3.80, p<0.00)$ test revealed that the male (mean rating 4.5) participants agreed with this statement to a greater extent than the female participants (mean rating 2.9), indicating that the males had greater confidence in their navigational ability. 


\subsection{Efficiency}

\subsubsection{Additional Nodes}

The number of additional nodes opened over and above the minimum needed to locate each answer was recorded for each subject. An independent samples t test $(F=0.74, p<0.40)$ revealed that there was no significant difference in the number of additional nodes opened by male $($ mean $=8.7)$ and female $($ mean $=7.7)$ participants.

\subsubsection{Accuracy}

The number of correct answers was recorded for each subject. An independent samples $t$ test $(F=1.9, p<0.183)$ revealed that there was no significant difference between male $($ mean $=9.4)$ and female participants $($ mean $=9.5)$ in the number of correct answers given.

\subsubsection{Navigation Strategy}

After each completed search participants were asked to indicate whether they had navigated through the system according to a specific navigational plan or had navigated according to situational navigational cues. Table 1 shows the total scores for males and females.

Table 1. Contingency table for navigational strategy choice

\begin{tabular}{|l|l|l|}
\hline & Planned & Situation-Specific \\
\hline Male & 74 & 26 \\
\hline Female & 40 & 60 \\
\hline
\end{tabular}

A Chi-Square test revealed that the choice of navigational strategy was related to gender $\left(\mathrm{df}=1, \mathrm{X}^{2}=23.58, \mathrm{p}<0.01\right)$. Males indicated that they had used a planned route through the system, whereas females relied upon situational navigation cues such as landmarks.

\subsubsection{Verbal Data}

During the direction-giving task participants were asked to direct another individual to a specified node in the web-site. The resulting verbal protocols were analysed for references to the use of landmarks during wayfinding and navigational decisions (see Tables 2 and 3 respectively). 
Table 2. Total number of comments made with regard to wayfinding

\begin{tabular}{|l|l|l|}
\hline Category & Males & Females \\
\hline $\begin{array}{l}\text { Landmark used to make navigational } \\
\text { decisions }\end{array}$ & 5 & 21 \\
\hline Landmark used as a navigational goal & 5 & 17 \\
\hline $\begin{array}{l}\text { Landmark recognised from a previous } \\
\text { encounter }\end{array}$ & 6 & 17 \\
\hline
\end{tabular}

Mann Whitney $U$ tests revealed that female participants used landmarks to guide navigational decisions $(Z=-3.06, p<0.002)$, used landmarks as navigational goals $(Z=-2.86, p<0.05)$, and recognised landmarks from previous encounters $(Z=-2.53, p<0.02)$, significantly more than male participants.

Table 3. Total number of comments made with regard to navigational decisions

\begin{tabular}{|l|l|l|}
\hline Category & Males & Females \\
\hline Definite action & 18 & 5 \\
\hline Casual Analysis & 7 & 20 \\
\hline Exploratory action & 19 & 5 \\
\hline
\end{tabular}

Mann Whitney $U$ tests revealed that male participants made more definite actions $(Z=-3.45, p<0.001)$, and made more exploratory actions $(Z=-3.78$, $\mathrm{p}<0.00)$ than females. However, females tended to engage in casual analysis more than males $(Z=-3.54, p<0.00)$.

\section{DISCUSSION}

The results show that in terms of user confidence males express a significantly higher degree of confidence than do females in their ability to navigate through the web and retrieve information. These findings support those of Devlin and Bernstein [5], Lawton et al. [4], and Schmitz [9], who also found males to have greater confidence than females in their ability to navigate. These results also seem to support the findings of previous studies of gender differences in computer anxiety/confidence that attribute female anxiety and poor confidence levels to restricted computer access and the poor availability of female oriented software [11]. However, it may be that females' poor confidence ratings may also reflect a general tendency of women to underestimate or belittle their performance of tasks that are gender typed as masculine [14]. For example, Beyer [14] found that women's confidence estimates were affected not only by their actual performance on masculine typed tasks but also by their own low expectations of their performance. According to Harris [15] and Lawton et al. [4] environmental wayfinding is stereotyped as a masculine activity. 
Despite their greater degree of confidence, there was no difference between males and females in terms of their navigational efficiency. These findings contrast with those of Lawton et al. [4] who found males to be more efficient than females. However, within the context of this study, navigation efficiency was measured by participants' ability to retrieve the answers to questions correctly following the most direct route. If task speed had been an efficiency measure then gender differences may well have emerged since the females tended to engage in a greater degree of casual analysis, pausing for thought more and spending considerably longer scanning individual pages looking for recognisable features such as graphics or table elements.

The results did show that there were striking differences in the way males and females navigated through the system. Females relied more heavily on the use of landmarks to guide their navigation, and indicated that they tended to react more to environmental cues rather than having a route plan in mind. Male participants however, stated that they navigated to a pre-planned route, not making as many references to landmarks as females.

The results of the verbal data support the findings that females make greater use of landmarks during navigation than male participants do. This supports previous studies of gender differences in real world navigation. The results also show that male participants were more definite about their movements through the system, and more often engaged in exploratory behaviour. Females however, were more likely to carefully think about an action and engage in what we call casual analysis than male subjects do. These findings also lend support to the confidence ratings data.

The results of this study have two broad implications. First, they suggest that parallels do exist between navigation in the real world and navigation in electronic space. Second, they indicate that while there were no gender differences in navigational efficiency, there are differences in navigational strategy. Therefore, as electronic spaces such as the web grow in size, complexity and use, there is a greater need to provide integrated support for these different strategies so that neither gender group is disadvantaged. Previous work into the development of navigational aids for hypertext systems such as the web have focused on the development of spatially based aids such as spatial maps, fisheye browsers, and graphical history trees. The results of the present study suggest that while these aids may help male users they may be of little or no benefit to female users who tend to rely upon landmarks to guide their navigation. Therefore, we need to identify ways in which we can leverage women's preference for landmarks; for example, through the use of coloured regions, distinctive landmark nodes or signposts within a site.

The results of this study suggest that gender differences in web navigation occur in user confidence and adopted navigation strategy. Further 
work is needed to study these differences in more detail, plus the kinds of navigational support mechanisms or design features to assist both male and female web users. Such work is currently underway at our laboratory.

\section{REFERENCES}

1. McDonald, S., \& Stevenson, R.J. (1998). The effects of text structure and prior knowledge of the learner on navigation in hypertext. Human Factors 40, (1), pp.18-27.

2. McDonald, S., \& Stevenson, R.J. (1998). Navigation in Hyperspace: an evaluation of the effects of navigational tools and subject matter expertise on browsing and information retrieval in hypertext. Interacting with Computers. 10(2), pp.129-142.

3. Tauscher, L., \& Greenberg, S. (1997). Revisitation patterns in World Wide Web navigation. ACM SIGCH'97 Conference on Human Factors in Computing Systems. Atlanta, Georgia: ACM Press.

4. Lawton, C.A., Charleston, S. I., and Zieles, A.S. (1996). Individual and gender-related differences in indoor wayfinding. Environment and Behaviour, 28(20), pp.204-219.

5. Devlin, A. S., and Bernstein, J. (1995). Interactive wayfinding - use of cues by men and women. Journal of Environmental Psychology, 15(1), pp.23-38.

6. Kim, H., \& Hirtle, S.C. (1995). Spatial metaphors and disorientation in hypertext browsing. Behaviour and Information Technology, 14, pp.239-250.

7. Dabbs, J.M., Chang, E.L., Strong, R.A., and Milun, R. (1998). Spatial ability, navigation strategy, and geographic knowledge among men and women. Evolution and Human Behaviour. 19 (2), pp.89-98.

8. Sandstrom, N. J., Kaufman, J., and Huettel, S.A., (1998). Males and females use different distal cues in a virtual environment navigation task. Cognitive Brain Research, 6(4), pp.351-360.

9. Schmitz, S. (1997). Gender-related strategies in environmental development: Effects of anxiety on wayfinding in and representation of a three-dimensional maze. Journal of Environmental Psychology. 17(3), pp.215-228.

10. Miyake, N. (1986). Constructive Interaction and the Iterative Process of Understanding. Cognitive Science. 10, pp.151-77.

11. Corston, R., and Colman, A.M. (1996). Gender and social facilitation effects on computer competence and attitudes towards computers. Journal of Educational Computing Research 14(2), pp.171-183.

12. Miller, L.K., \& Santoni, V. (1986). Sex differences in spatial abilities: Strategic and experiential correlates. Acta Psychologica, 62, pp.225-235.

13. Galea, L.A.M., \& Kimura, D. (1993). Sex differences in route learning. Personality and Individual differences 14, pp.53-65.

14. Beyer, S. (1990). Gender differences in the accuracy of self-evaluations of performance. Journal of Personality and Social Psychology, 59, pp.960-970.

15. Harris, L.J. (1981). Sex-related variations in spatial skill. In L.S. Liben, A.H. Patterson, \& N. Newcombe (Eds.) Spatial Representation and Behaviour Across the Lifespan (pp.83125) New York: Academic Press. 